\title{
Gathering Usage Statistics for E-Resources at Wheaton College
}

\author{
by Megan M. Ruenz
}

Usage statistics are an important part of the evaluation process for e-resources of any type: journals, e-books, or databases. Providing insight into how resources are used, usage statistics can be helpful in making decisions on whether to renew a resource. Usage reports can also show a library's value to the greater campus and may even be reported in the library's annual report.

The e-resources department at Buswell Library gathers usage statistics from many vendors in order to evaluate our e-resources. Statistics that are reported in COUNTER (Counting Online Usage of Networked Electronic Resources) format are the most desirable because these statistics are the most comparable. The COUNTER organization provides a code of practice that ensures all publishers and vendors report the usage of their electronic resources in a consistent, credible, and comparable way. The most recent release of COUNTER statistics is the Release 5, and one of the major enhancements that our library has seen with Release 5 is with the "Unique" metric that removes duplication and inconsistencies in reporting.

Not every publisher can provide COUNTER statistics, so, in those cases, Buswell Library reports the non-COUNTER statistics from publishers. These types of statistics include metrics like tracks of a sound recording played and plays viewed of a streaming video title. Since these metrics may not be exactly the same as other metrics that are reported, they must be converted to COUNTER-like statistics as closely as possible to be comparable.

Until Buswell Library migrated to Alma, gathering usage statistics was quite an involved and manual process. Library staff visited every vendor website to gather statistical reports of interest, which we totaled for each publisher/vendor and then manually entered into a master spreadsheet for each report type (TR_J1, TR_B1, DR_D1, and non-COUNTER). The TR_J1 report shows the usage of journal content, excluding gold open access content, on a publisher's platform. For usage data related to the number of times an e-book is used on a publisher's platform, the TR_B1 report is the COUNTER report for that material. A DR_D1 report measures the number of database searches. Non-COUNTER reports report the usage in non-COUNTER format, meaning the publisher has their own way of recording usage. The master spreadsheet that Buswell Library enters its usage data into was then used to report statistics for the library's annual report. Because of the time-intensity of this manual process, gathering usage statistics was only done on demand or around the time that the annual report was prepared. Our department just didn't have the staffing resources to gather statistics more frequently.

The e-resources department moved to storing vendor administrative credentials in LastPass in 2019. We previously used CORAL, an open-source electronic resources management tool, for this information, but as we found ourselves using CORAL less frequently in our daily workflow, the decision was made to switch this information over into LastPass. The LastPass interface is easy to use and allows folders of credentials to be shared with others in the department as needed.

In early 2021, the e-resources librarian was able to hire a student worker to help with the gathering of usage statistics while this process was still manual. The student was given access to a shared LastPass folder that housed the vendor administrative credentials and then was trained to retrieve the desired reports. After retrieving reports, the student worked with the data and entered the

Megan Ruenz is the E-resources Librarian at Wheaton College. 
information into the master spreadsheet. Having a student do this work more regularly made this data more readily available to our librarians.

A migration to Alma in the summer of 2020 changed the way we gather usage statistics. During our first year using Alma, we continued to gather usage statistics in the same manner as described above, but, in late spring of 2021, we started to explore how we could use Alma to gather our usage data. Alma has SUSHI (Standardized Usage Statistics Harvesting Initiative) capabilities built into it that allow for the automatic harvesting of usage statistics. The SUSHI server sends an automated request to the publisher for COUNTER reports over a range of several months. Once Alma harvests the usage statistics, the COUNTER reports can then be viewed using Alma Analytics. While there was quite a bit of work involved in setting up SUSHI harvesting in Alma, it has proven to be beneficial to have all of our data in one place to run other analytics as our library needs. To set up SUSHI in Alma, we visited each vendor's administrative portal to get their SUSHI credentials. In some cases, SUSHI credentials can be retrieved directly from the vendor administrative portal with nothing further to do for setup, while other vendors require librarians to contact their customer service department in order to create or distribute SUSHI credentials for use. Once we know the credentials, we could enter them into the vendor record in Alma along with the vendor server URL. The final SUSHI setup in the Alma vendor record is to select the reports for Alma to harvest. Alma allows the ability to test the connection with the vendor server before harvesting reports, which is a great feature to have built into the interface. Once all the information is in the Alma vendor record (figure 1), the last step is to set how often the usage data will be harvested. Buswell Library harvests monthly since vendors rarely make changes to their usage data mid-month, but data can be harvested weekly if desired.

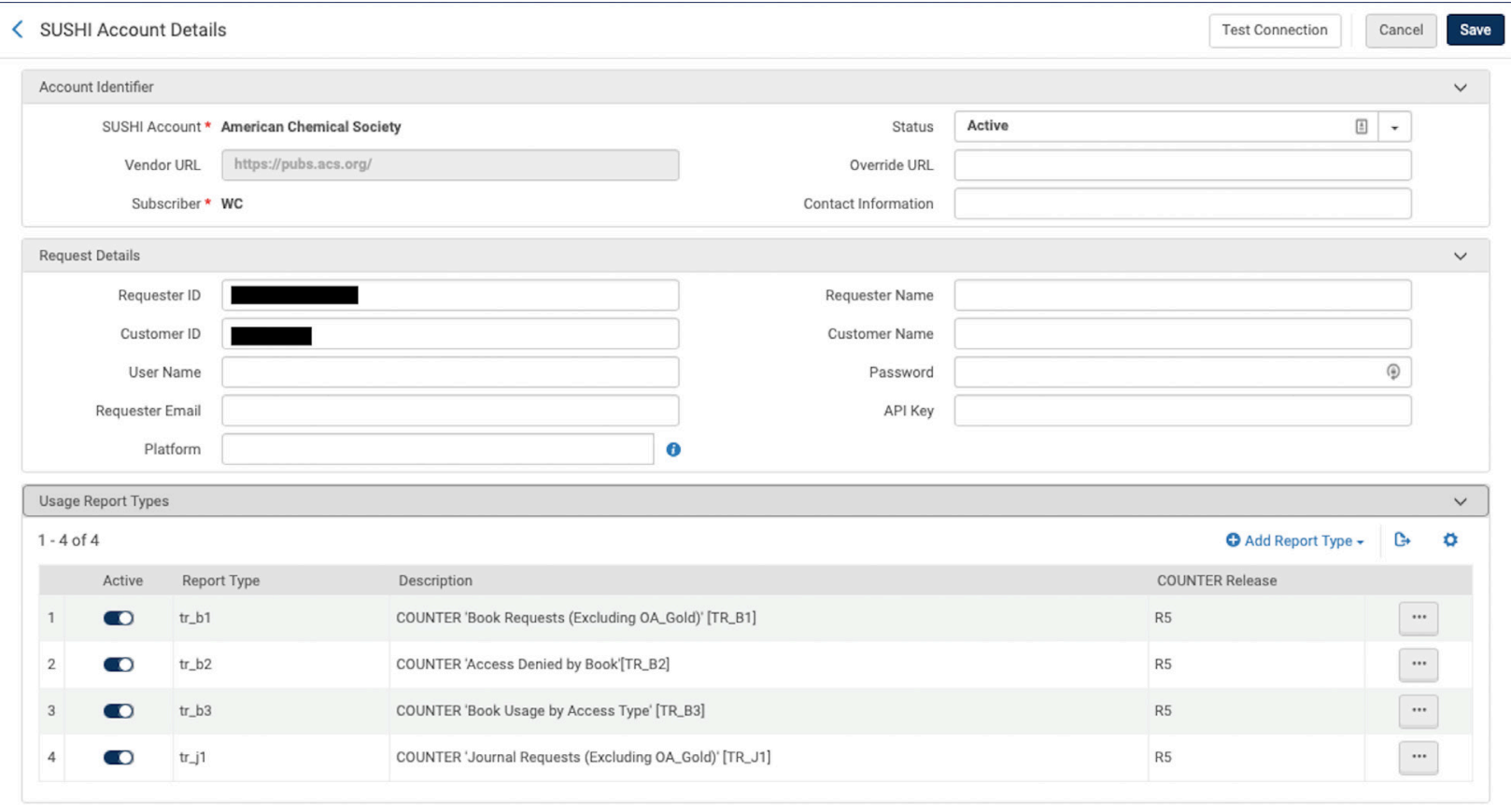

Figure 1: SUSHI setup in Alma Vendor record 
Once Alma has harvested the COUNTER reports, they can be viewed in the "Usage Data" tab of the vendor record. In the section titled "Uploaded Files," the reports that have been harvested by SUSHI or uploaded manually (figure 2) are visible. Alma retrieves usage data files in JSON format, which will need to be converted if you wish to view the contents of the report. Alma will indicate the status of each report harvested, letting you know if it was fully processed, partially processed, or if it wasn’t processed at all.

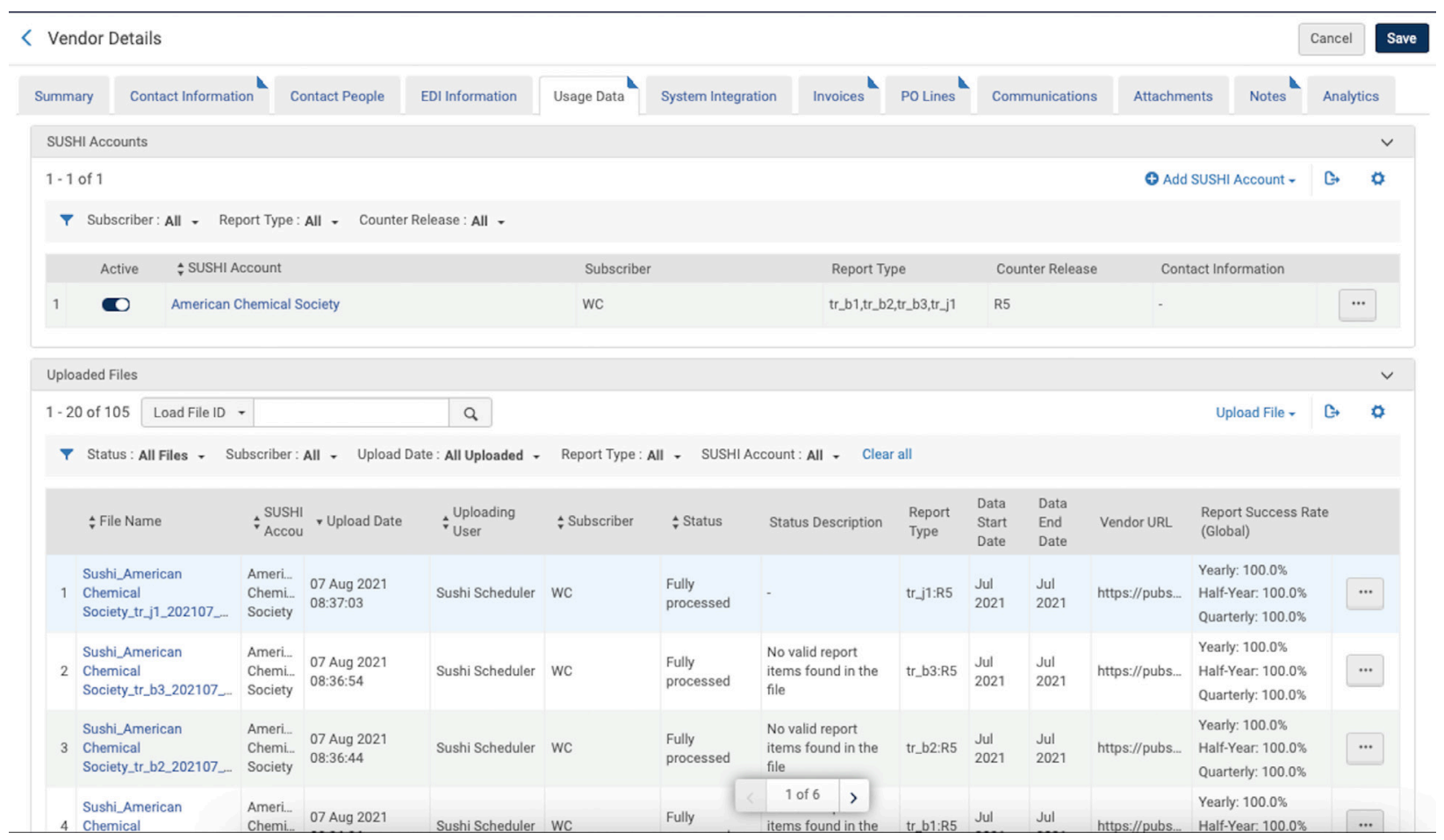

Figure 2: Harvested usage reports in Alma

Even though Alma does not automatically harvest non-COUNTER reports, this data can be loaded into Alma as well. To load these reports into Alma, the usage data first needs to be put into a COUNTER-like format so that it can be recognized and compared alongside the COUNTER data. To make these reports COUNTER-like, the usage data is converted to that of an article download or a database session, depending on the usage data being collected. The e-resources librarian at Buswell Library makes that decision on a case-by-case basis. Once the reports are uploaded into Alma, these too can be viewed in the same section as the SUSHI-harvested reports shown in figure 2.

In addition to making sure the usage reports have been uploaded into or harvested from Alma, the usage data in these reports can be viewed in Alma Analytics. There are many templates and shared reports in the Alma Analytics community, but reports can also be customized for the needs of each individual library. In the Alma Analytics community, I found templates for reports that showed monthly usage and yearly usage. These reports were customized to add the information that was relevant to our needs (figure 3). Analytics reports can be viewed on screen or downloaded in a more workable format (.xls, .pdf, .ppt, .csv, .txt, or .xml). Buswell Library also uses Alma to manage our acquisitions data, and this allows me to use analytics to run cost-per-usage data with 
our COUNTER and COUNTER-like data. One full year after migration, I am beginning to learn how powerful and capable Alma Analytics reporting can be.

For libraries that are not using Alma, there is another option to harvesting usage statistics with ease using SUSHI. Melissa Belvadi, systems librarian at the University of Prince Edward Island,

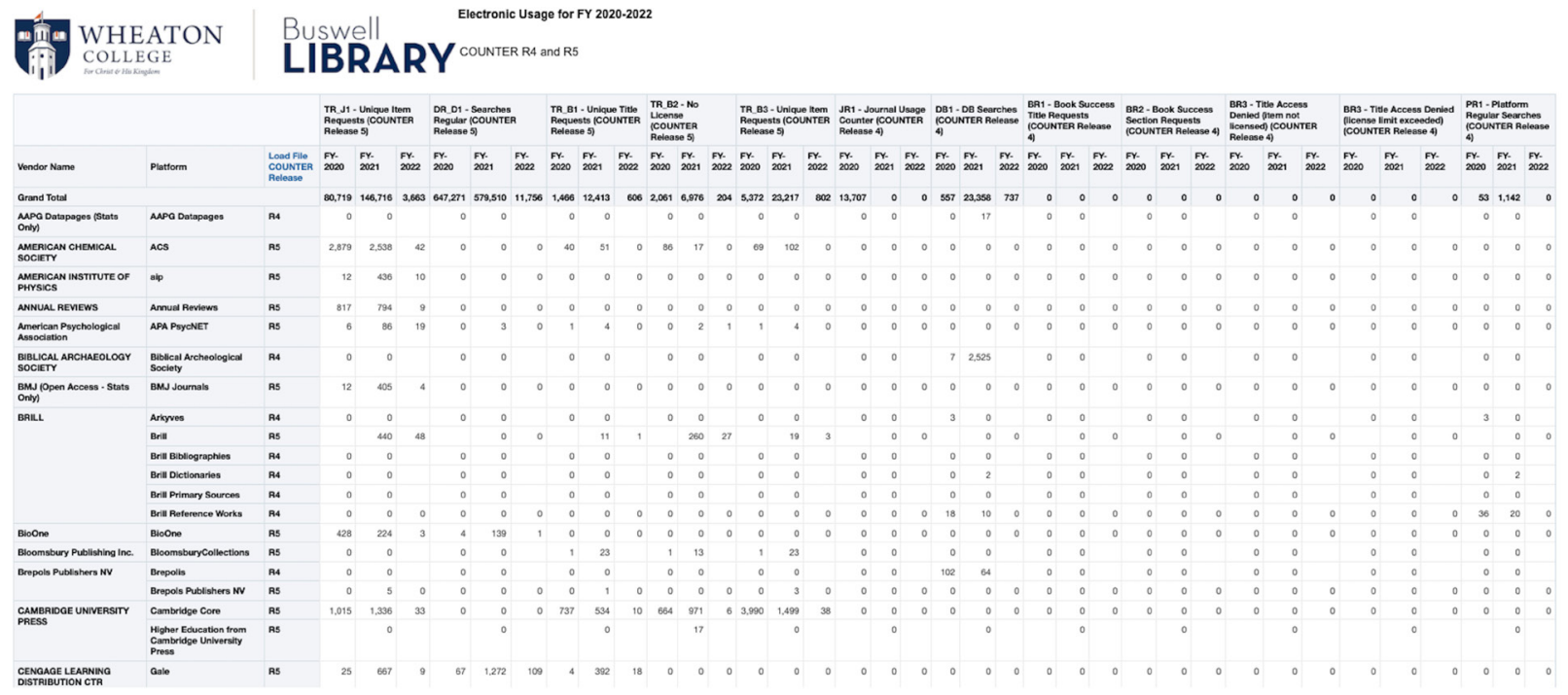

Figure 3: Alma Analytics report from SUSHI-harvested data and manually uploaded COUNTER reports

developed a COUNTER 5 Reporting Tool with a group of senior-level computer science students in 2019. This reporting tool works entirely on a local desktop and was designed to give individual libraries a convenient way to harvest their usage data. The COUNTER 5 Report Tool gathers usage statistics by using the same vendor SUSHI credentials and connecting to the vendor's SUSHI server. All the vendor information and SUSHI credentials are stored in the Manage Vendor tab (figure 4) where the vendor information entered into the fields for each vendor will be used to fetch desired reports. The Manage Vendor tab allows users to check the non-SUSHI box if the desire is to use this tool as a repository for vendor credentials. To share vendor information with others, there is an option to export the vendor information into a file where it can later be shared. In the Fetch Reports tab (figure 5), the user selects the vendor(s) and report(s) they'd like to run along with the date range for the desired reports. The COUNTER 5 Reporting Tool connects to the servers of each vendor selected and returns the usage data results in an XLS file in a specified folder on your computer. This makes the data easy to find and very easy to work with. One of the best features of this tool is how quickly the reports are gathered. In my own experience, this tool retrieved almost forty COUNTER reports from various vendors in under ten minutes. The COUNTER 5 Reporting Tool can also be used for cost analysis when financial information is entered into the tool.

Gathering usage statistics is a necessary part of evaluating our library's electronic resources. Since our library's migration to Alma, gathering usage statistics has become a more automated process which will allow us the opportunity to review and analyze our data more efficiently. 


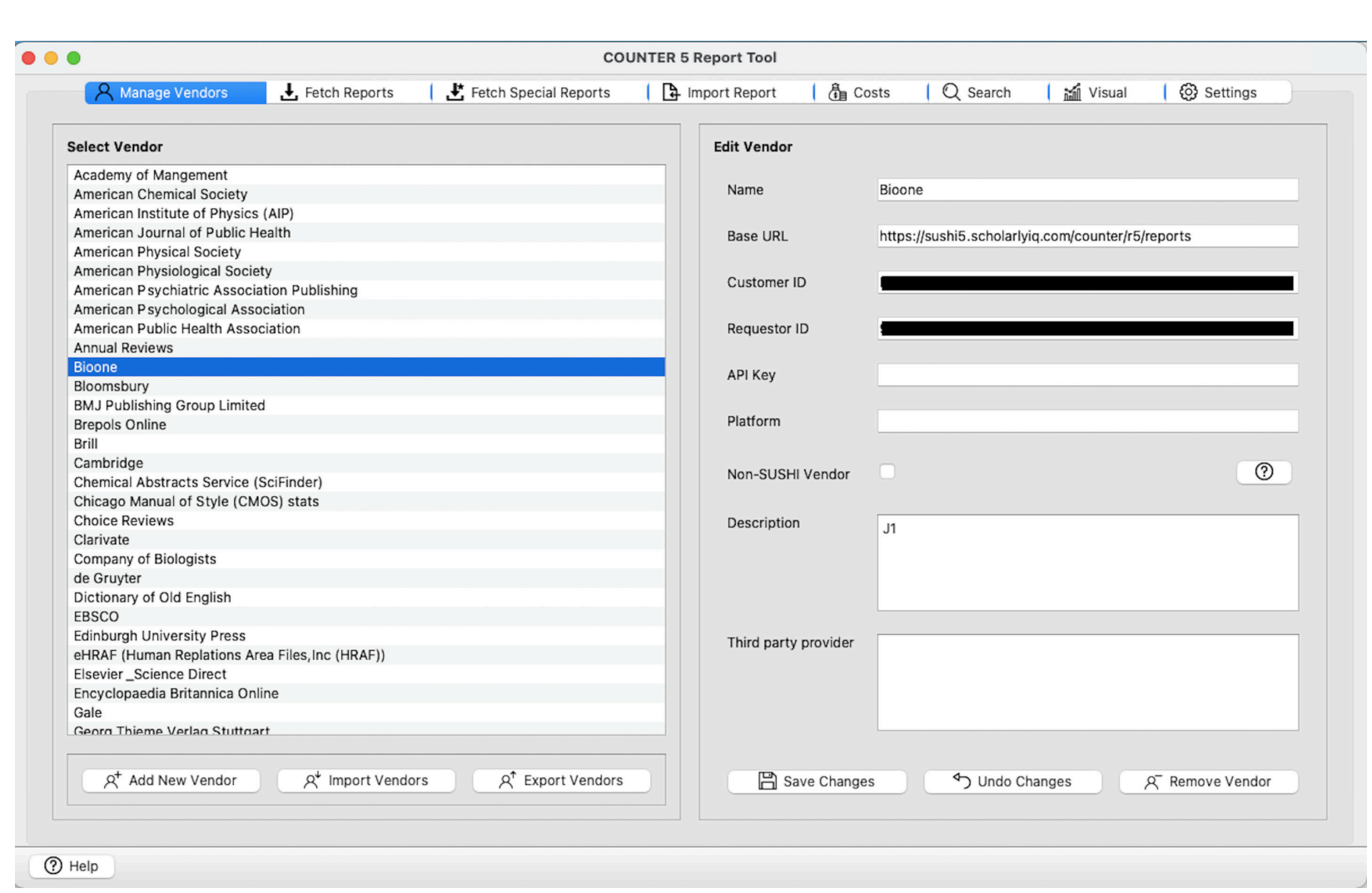

Figure 4: SUSHI setup in the Manage Vendor Tab of the COUNTER 5 Reporting Tool

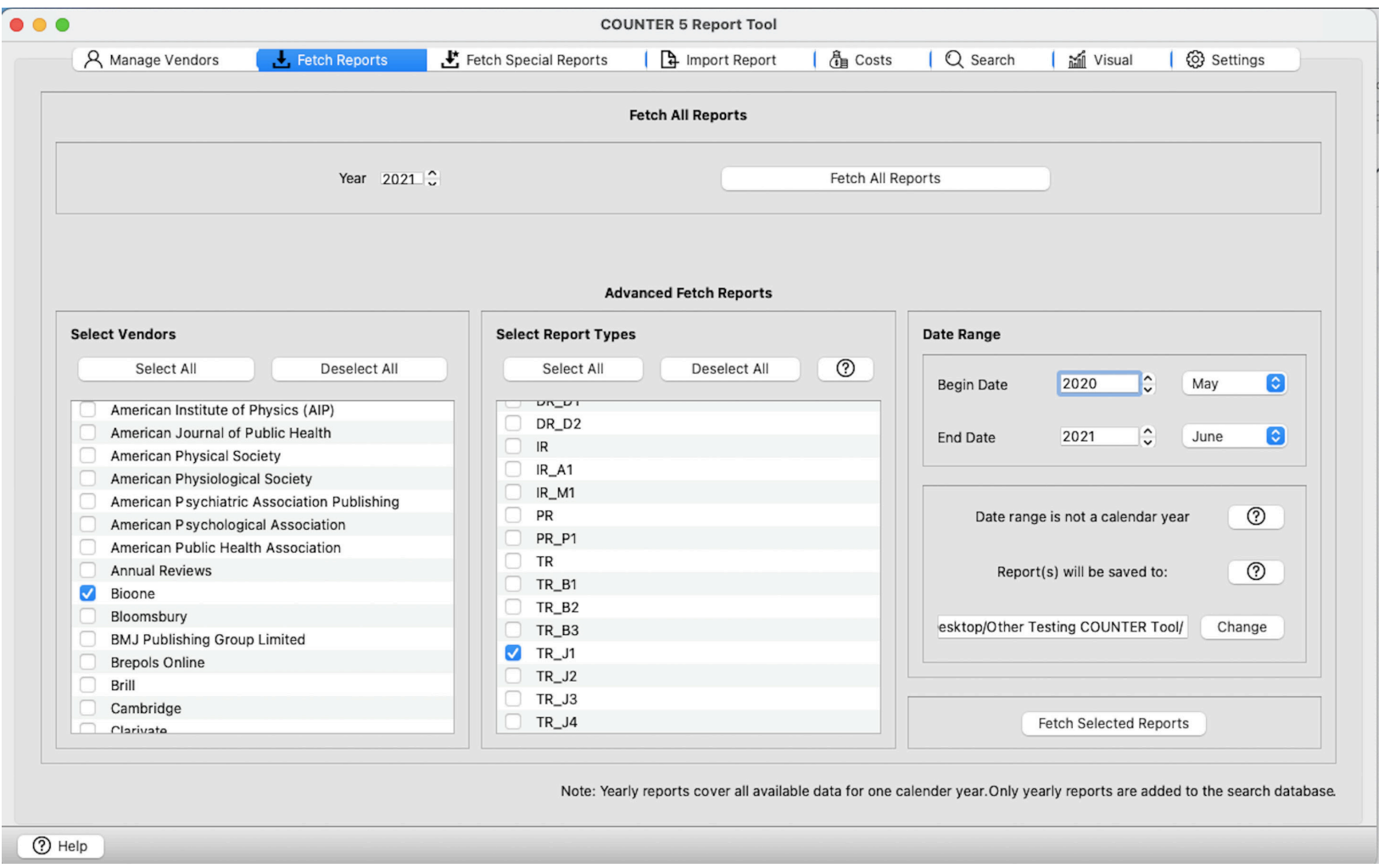

Figure 5: Running reports in the Fetch Reports Tab of the COUNTER 5 Reporting Tool 